\title{
Glass Transitions in Monodisperse Cluster-Forming Ensembles: Vortex Matter in Type-1.5 Superconductors
}

\author{
Rogelio Díaz-Méndez, ${ }^{1}$ Fabio Mezzacapo, ${ }^{1}$ Wolfgang Lechner, ${ }^{2}$ Fabio Cinti, ${ }^{3,4}$ Egor Babaev, ${ }^{5}$ and Guido Pupillo \\ ${ }^{1}$ icFRC, IPCMS (UMR 7504), ISIS (UMR 7006), Université de Strasbourg and CNRS, 67000 Strasbourg, France \\ ${ }^{2}$ IQOQI and Institute for Theoretical Physics, University of Innsbruck, 6020 Innsbruck, Austria \\ ${ }^{3}$ National Institute for Theoretical Physics (NITheP), Stellenbosch 7600, South Africa \\ ${ }^{4}$ Institute of Theoretical Physics, Stellenbosch University, Stellenbosch 7600, South Africa \\ ${ }^{5}$ Department of Theoretical Physics and Center for Quantum Materials, KTH-Royal Institute of Technology, \\ Stockholm SE-10691, Sweden
}

(Received 2 May 2016; published 8 February 2017)

\begin{abstract}
At low enough temperatures and high densities, the equilibrium configuration of an ensemble of ultrasoft particles is a self-assembled, ordered, cluster crystal. In the present Letter, we explore the out-ofequilibrium dynamics for a two-dimensional realization, which is relevant to superconducting materials with multiscale intervortex forces. We find that, for small temperatures following a quench, the suppression of the thermally activated particle hopping hinders the ordering. This results in a glass transition for a monodispersed ensemble, for which we derive a microscopic explanation in terms of an "effective polydispersity" induced by multiscale interactions. This demonstrates that a vortex glass can form in clean systems of thin films of "type-1.5" superconductors. An additional setup to study this physics can be layered superconducting systems, where the shape of the effective vortex-vortex interactions can be engineered.
\end{abstract}

DOI: 10.1103/PhysRevLett.118.067001

The vortex glass is one of the key states in the theory of magnetic and transport properties of type- 2 superconductors in the presence of disorder [1-4]. Within this frame, the glassy phase is caused by the pinning of vortices by impurities and is absent in a clean sample. In this work, we demonstrate that a vortex glass state can be an inherent property of a superconducting system characterized by multiple coherence lengths. In a more general context, our work demonstrates that a structurally disordered glass state of matter can be obtained in the absence of disordered substrates for a simple two-dimensional monodisperse ensemble of particles interacting via isotropic, repulsive, ultrasoft interactions [5,6]. This is surprising as those conditions are usually associated with minimal frustration [7-10]. The glass phase appears below a nonequilibrium glass transition temperature and extends to the lowest temperatures examined. We provide a description of the microscopic mechanism responsible for the appearance of glassiness in terms of an effective polydispersity that emerges following a quench due to multiscale interactions [see Fig. 1]. In typical glass forming liquids, frustration results from polydisperse mixtures of particles [11]. In the present systems, the appearance of glassiness stems from the effective polydispersity of cluster sizes.

Several works have recently discussed "type- 1.5 superconductors" that are characterized by multiple coherence lengths, some of which are larger and some smaller than the magnetic field penetration length. These multiple coherence lengths arise in superconducting states that break multiple symmetries and, also, in materials with multiple superconducting bands. Several materials were suggested in experiments to belong to this type of superconductors [12-15], where vortices can display multiscale attractive and repulsive intervortex interactions [12,14,16-18]. Multiple attractive length scales come from core-core intervortex interactions. Multiple repulsive length scales can be obtained, instead, in (i) artificially fabricated superconducting bilayers, where the different layers give rise to two coherence lengths, or rather generally in (ii) thin films of type-1.5 materials due to stray fields.

In the case of artificial superconducting bilayers [case (i) above], the London's magnetic field penetration length will, in general, be different in the different layers. In this case, cocentered vortices form in different layers in the presence of a perpendicular magnetic field. When the interlayer electromagnetic coupling is strong (or there is substantial interlayer proximity effect), fluctuations associated with the loss of axial symmetry of vortices can, under certain conditions, be neglected. For a sufficiently high vortex line tension, a dilute system of such vortices can be mapped onto point particles with the following intervortex interaction potential at long-ranges derived in the Supplemental Material [19]:

$$
U(r)=\sum_{i=1,2}\left[C_{B_{i}}^{2} K_{0}\left(\frac{r}{\lambda_{i}}\right)-C_{i}^{2} K_{0}\left(\frac{r}{\xi_{i}}\right)\right] .
$$

Here, $K_{0}$ is the modified Bessel function of the second kind. In the Supplemental Material [19], we show 
simulations for such a system with different London's magnetic field penetration lengths $\lambda_{1,2}$, coherence lengths $\xi_{1,2}$, and coefficients $C_{B_{i}}$ and $C_{i}$, which are weakly dependent on $T$ (see Supplemental Material [19]). The resulting potential shape for that particular choice is shown in Fig. 1(a) with a blue dashed line.

In the case of films of type-1.5 superconductors [case (ii) above] the long range interaction potential acquires a term that decays with distance as $1 / r$ due to Pearl's effect [36]

$$
U(r)=C_{B}^{2} K_{0}\left(\frac{r}{\lambda}\right)+\frac{A}{r}-\sum_{i} C_{i}^{2} K_{0}\left(\frac{r}{\xi_{i}}\right) .
$$

Here, as in the ordinary films of type- 2 superconductors, we separated the electromagnetic interaction in the interior of the film and the Pearl's $A / r$ correction arising from demagnetization fields ( $A$ being a constant). The prefactors $C_{B}$ and $C_{i}$, in Eq. (2) depend on the film thickness, while $\lambda$ and $\xi_{i}$ depend on the choice of material. Both potentials in Eqs. (1) and (2) [thin dashed and thick blue curves in Fig. 1(a), respectively] are two-scale repulsive, in contrast to the single-scale repulsive intervortex potentials in usual type- 2 superconductors. In their range of validity, they have a "plateaulike"
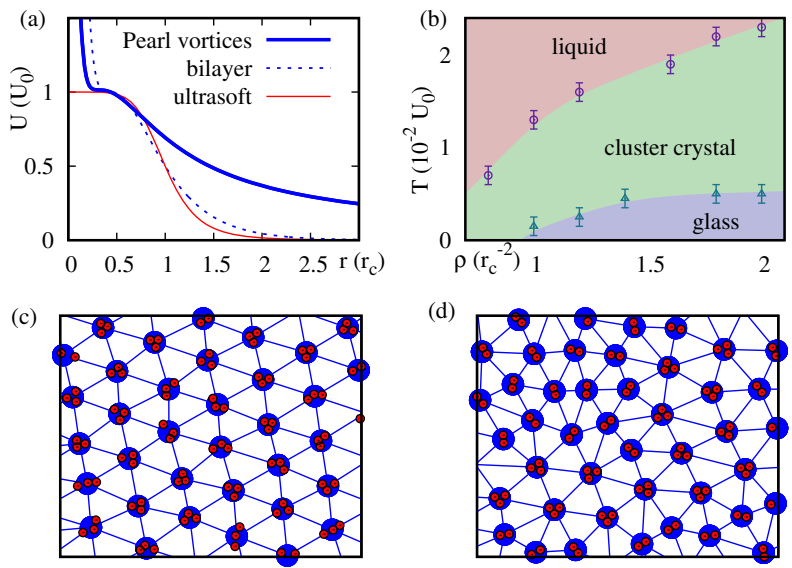

FIG. 1. (a) Cluster-forming interaction for a system of vortices in 1.5 superconducting films [thick blue line, Eq. (2) in the text]. Also shown is the interaction potential for vortices in 1.5 bilayer superconductors [dashed blue line, Eq. (1) in the text], and the generic ultrasoft potential [red line, Eq. (3) in the text]. (b) Dynamic phase diagram of the model of Eq. (2) as a function of rescaled density and temperature. Circles and triangles indicate the liquid-to-crystal and the glass transition temperatures, respectively. (c) Snapshot of a crystal configuration after quenching a monodisperse vortex system with the potential Eq. (2) [blue line in panel (a)], for density $r_{c}^{2} \rho=1.6$ at temperature $T=1.8 \times 10^{-2} U_{0}$. Single vortices (red circles) group into clusters (blue circles), blue lines join nearest neighboring clusters as obtained by Delauney triangulation. (d) Same as (c) for the glass phase at density $r_{c}^{2} \rho=1.6$ and temperature $T=$ $0.2 \times 10^{-2} U_{0}$. In all simulations, we choose the values $A / U_{0}=0.7364, \quad C_{B}^{2} / U_{0}=8.124, \lambda / r_{c}=0.0084, \quad C_{1}^{2} / U_{0}=$ 0.884 , and $\xi_{1} / r_{c}=0.238$. feature of value $U_{0}$ at intermediate distances that extends up to a distance $r \simeq r_{c}$, and then decays for $r \gtrsim r_{c}$. Note that, for type-1.5 vortices, one can have a more pronounced minimum in the place of the plateau which yields similar results. Here, we are interested in the dynamics of two-dimensional vortices following a quench from an initial high-temperature to final low-temperature $T$, for vortex densities $\rho$ such as $r_{c}^{2} \rho \gtrsim 1$. We perform molecular dynamics simulations with an overdamped Langevin thermostat of friction coefficient $\gamma$, governed by the equation $\dot{\vec{r}}=-\nabla U / \gamma+\sqrt{2 k_{B} T / \gamma} \vec{\eta}(t)$, where $\vec{\eta}(t)$ is a Gaussian stochastic force with zero mean and unit variance and $k_{B}$ is the Boltzmann constant [37-39]. The units of length, time, temperature, and density are $r_{c}$, $\gamma^{-1}, U_{0}$, and $r_{c}^{-2}$, respectively, and the total number of particles $N$ varies from $N=600$ to 15000 [40].

Figure 1(b) shows the phase diagram of Eq. (2) following the temperature quench to a final value $T$ [see below for details on observables]. For comparatively large $T$ the system remains in a liquid phase, while for intermediate temperatures, it equilibrates, and the resulting phase is a cluster crystal [see snapshot in panel (c)]. In this phase, particles group into clusters, which, in turn, are ordered in a triangular lattice with approximately the same number of particles per site. For the lowest temperatures, we find, instead, a surprising lack of equilibration that keeps the system in a disordered configuration [see panel (d)]. The demonstration of a resulting vortex glass in the absence of substrate disorder and its microscopic explanation in terms of effective polydispersity (see below) are main results of this work.

We note that, by a further increase of the density with $\rho r_{c}^{2} \gg 1$, the low- $T$ configuration in both models above can evolve through states induced due to multiple interaction scales. At equilibrium, these do not resemble simple triangular cluster crystals. As an example, the formation of a disordered nematiclike phase for the bilayer model of Eq. (1) and density $r_{c}^{2} \rho=4.0$ is shown in the Supplemental Material [19].

Since the divergence of the interaction potential at $r=0$ is an artefact of asymptotical analysis, in the following, we also consider a model of cluster-forming potential where the unphysical short-range divergence is removed. We come back to the potentials of Eqs. (1) and (2) in the Supplemental Material [19]. This model potential reads

$$
U(r)=U_{0}\left[1+\left(r / r_{c}\right)^{6}\right]^{-1} .
$$

Such a potential approaches the constant value $U_{0}$ as the interparticle distance $r$ decreases below the soft-core radius $r_{c}$, and drops to zero for $r>r_{c}$ as $r^{-6}$, i.e., with a repulsive van der Waals tail [43]. Ultrasoft potentials of this kind have recently attracted considerable attention [44-47] as mean-field approximations of interpolymer interactions in soft-matter systems as diverse as dendritic polymers, polymer rings, and chains. Because of their negative 
Fourier components [48-50], they provide an unexpected route towards self-assembly of composite crystalline structures for sufficiently large densities.

The phase diagrams for all models following a temperature quench are determined by computing both static and dynamical observables, corresponding to the hexatic order parameter for clusters $\Psi_{6}=\left\langle\sum_{j}^{N_{c}} \sum_{l}^{N_{j}} e^{i 6 \theta_{j l}} /\left(N_{c} N_{j}\right)\right\rangle$ (see Ref. [51]), the static structure factor $S(\mathbf{k})=$ $\left\langle\left|\sum_{j}^{N} e^{i \mathbf{k} \cdot \mathbf{r}_{j}}\right|^{2} / N\right\rangle$, the mean-square displacement $\left\langle\Delta r^{2}(t)\right\rangle=\left\langle\sum_{j}\left|\mathbf{r}_{j}(0)-\mathbf{r}_{j}(t)\right|^{2}\right\rangle / N, \quad$ and the nonGaussian parameter $\alpha_{2}(t)=\left[\left\langle\Delta r^{4}(t)\right\rangle /\left(2\left\langle\Delta r^{2}(t)\right\rangle^{2}\right)-1\right]$. Here, angular brackets $\langle\cdot\rangle$ denote an average over quench experiments, $N_{c}$ is the total number of clusters, $N_{j}$ is the coordination number of cluster $j$ (corresponding to the number of clusters neighboring the $j$ th one), $\theta_{j l}$ is the angle between a reference axis and the segment joining the clusters $j$ and $l$ (see Supplemental Material [19] and Ref. [51]), and $t$ is time.

Figure 2 shows results for the ultrasoft model of Eq. (3). Panel (a) shows the values of the hexatic order parameter $\Psi_{6}$ at equilibrium (red dashed line, see Ref. [51]), and after a quench (blue triangles) as a function of the final temperature $T$ of the system, for a fixed density. The equilibrium results display a single sudden jump of $\Psi_{6}$ from 0 to about 0.8 at $T_{c} \simeq 8 \times 10^{-2} U_{0}$, followed by a slow rise to 1 with decreasing $T$. This jump corresponds to a transition from the high-temperature disordered liquid to an ordered clustercrystalline phase for $T<T_{c}$. Each cluster here comprises the same time-averaged number of particles. The finite value of $S(\mathbf{k})$ in this finite system reflects the quasilong range order of
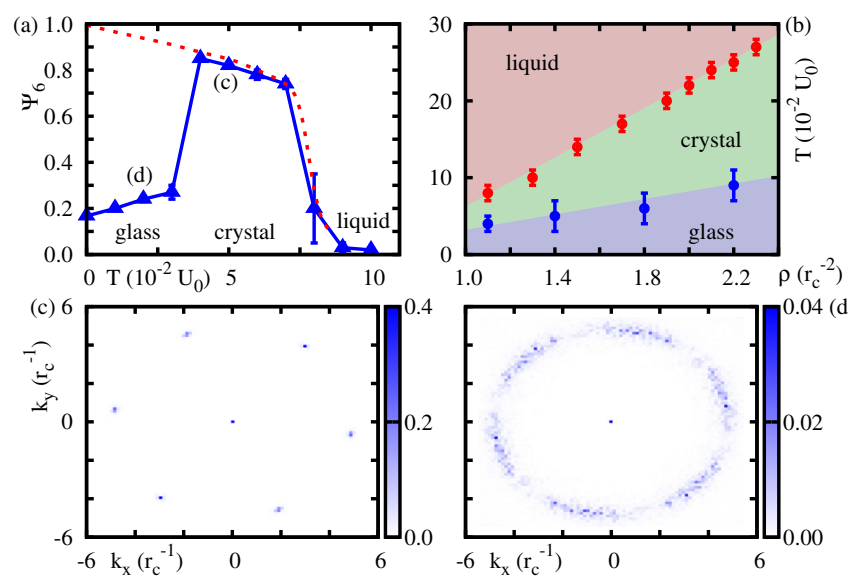

FIG. 2. (a) Hexatic order parameter $\psi_{6}$ as a function of the temperature for a system of particles interacting via the potential in Eq. (3) at equilibrium, and after a quench from high $T$ (red dashed line and blue triangles, respectively). The chosen value of the particle density is $r_{c}^{2} \rho=1.1$. (b) Dynamic phase diagram of the model as a function of rescaled density and temperature. Red and blue symbols indicate the liquid-to-crystal and the glass transition temperatures, respectively. Typical structure factors of the system are shown for the crystalline [panel (c)] and disordered glass [panel (d)] phases. the crystal. In contrast, the results following the temperature quench display two jumps. The first, at $T_{c} \simeq 8 \times 10^{-2} U_{0}$, corresponds to the onset of crystal formation: For $T_{g}<T<$ $T_{c}$ equilibration into a regular cluster crystal occurs in a time scale much smaller than the simulation time, and the crystal is essentially indistinguishable from the equilibrium situation [see Figs. 2(a) and 2(c)]. Conversely, the second jump is characteristic of the quenched dynamics and corresponds to the onset of glass formation: Below a characteristic temperature $T_{g}$, the value of $\Psi_{6}$ suddenly becomes small, signaling disorder. Disappearance of structural order is further demonstrated by the formation of a ringlike feature in $S(\mathbf{k})$ [Fig. 2(d)]. By inspection (see below), we find that disorder here results from the loss of ergodicity and the consequent lack of equilibration within the simulation time: Following the quench, particles quickly rearrange in clusters; however, particle hopping between clusters is suppressed so that the distribution of particles among the clusters remains disperse in time. This suggests that intercluster interactions, which depend on cluster occupancies, can vary significantly in the ensemble. As a result, clusters do not evolve into a large isotropic crystalline structure below $T_{g}$.

The emerging picture is one where an effective polydispersity of the clusters is realized in this low-temperature regime, corresponding to the formation of clusters with different occupancies. Our interpretation of the microscopic mechanism of induced polydispersity is quantified in the data of Fig. 3(b). The latter presents histograms of the measured coordination numbers of the clusters (i.e., the number of clusters which are nearest neighbor of a given cluster) for different cluster occupation values (i.e., number of particles in a cluster). The figure shows that the smaller the cluster occupation, the higher is the probability for a given cluster of being low coordinated, and vice versa. In other words, small "less repulsive" clusters are more likely to have less neighbors than large "more repulsive" ones. This strongly suggests a correlation between the induced "effective polydispersity" and the structural disorder of the glassy phase. An analogy can be drawn, here, with the effect of particle size distribution in the formation of disordered structures in genuinely polydisperse ensembles [11,52-56]. Interestingly, the equilibrium counterpart of this glass is a crystal, which turns into a glass for quenches at target temperature lower than $T_{g}$. We note that glass transitions have been previously found as a function of the degree of polydispersity in certain quasi-two-dimensional samples of binary colloidal suspensions [57]. The development of glassy properties in those polydisperse models is, in some respects, similar to the behavior found in our monodispersed ensemble, though here, glassiness originates solely from the multiscale interactions.

We confirm the glassy dynamics by monitoring timedependent observables such as the mean square displacement $\left\langle\Delta r^{2}(t)\right\rangle$ and $\alpha_{2}(t)$. Example results for $r_{c}^{2} \rho>1$ are shown in Fig. 3(a). In the figure, a linear dependence of 

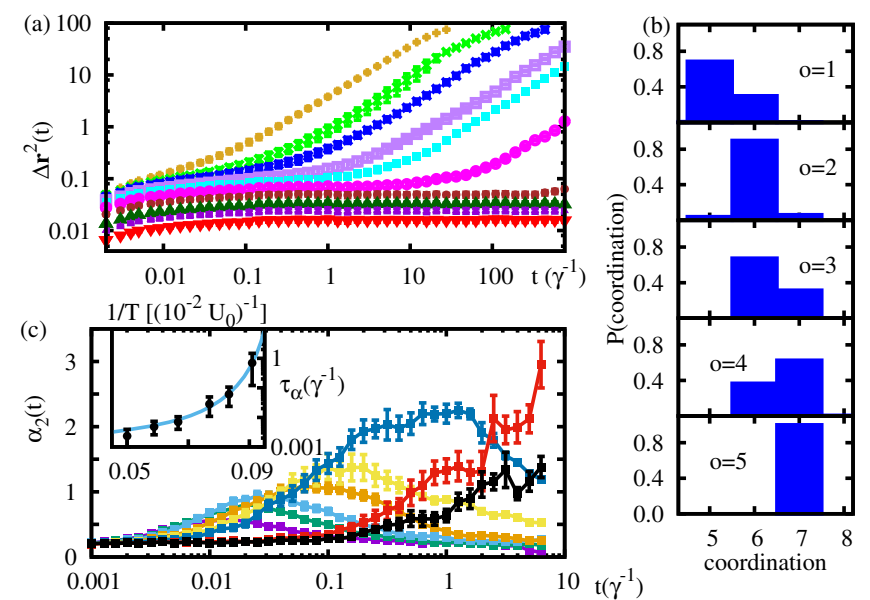

FIG. 3. (a) Mean square displacement for a system of particles interacting via Eq. (1) with $r_{c}^{2} \rho=1.1$, and temperatures, from top to bottom, $T=\{10,9,8, \ldots, 1\} \times 10^{-2} U_{0}$. (b) Probability distribution of the coordination number (i.e., the number of clusters nearest neighboring a given one) for subsets of clusters having the same occupation number $o$ (i.e., the number of particles in the cluster), for a choice of parameters so that the system is a glass. (c) Non-Gaussian parameter $\alpha_{2}(t)$ for density $\rho=1.4$. The temperatures (from the most left to the most right position of the maximum) are $T=\{12,11,9,8,7,6,5,3\} \times 10^{-2} U_{0}$. The inset shows the Arrhenius plot of the relaxation time, as extracted from the maximum of the $\alpha_{2}(t)$ parameter.

$\left\langle\Delta r^{2}(t)\right\rangle$ on time $t$ signals liquid behavior, which is evident for high $T$ (yellow line). For intermediate temperatures, however, $\left\langle\Delta r^{2}(t)\right\rangle$ develops a plateau at intermediate times. The latter is usually associated to caging effects when, close to a glass transition, mobility of individual particles is increasingly limited. In our case, this behavior occurs in the intermediate temperature range where the system rearranges in a cluster crystalline configuration. Here, the long-time liquidlike dynamics corresponds to residual activated particle hopping between the cluster sites, as observed first in Ref. [51]. Interestingly, for lower temperatures, the dynamics after a quench is completely arrested [i.e., $\left\langle\Delta r^{2}(t)\right\rangle$ takes a low value essentially constant in $t$ ], consistent with a transition to a glassy phase.

In Fig. 3(c), we plot the non-Gaussian parameter $\alpha_{2}(t)$ as a function of $t$ and for several values of $T$. This parameter measures deviations from Gaussian fluctuations in the distribution of displacements and, thus, is, in general, $\alpha_{2}(t)=0$ for all $t$ in regular liquids and noncluster crystals at equilibrium. Here, at intermediate temperatures, $\alpha_{2}(t)$ takes a maximum for a characteristic time $t=\tau_{\alpha}$. This signals the presence of different time scales usually associated with dynamical heterogeneity and out-ofequilibrium glassy dynamics. Our estimates for $\tau_{\alpha}$ are consistent with a Vogel-Fulcher-Tamman dependence on temperature [inset in Fig. 3(c)], which, within the structural glass-forming liquid scenario, usually indicates a fragile nature of the glass transition. The glass phase is found to extend down to the lowest temperatures probed. In the Supplemental Material [19], we show that the energy barriers that prohibit hopping between clusters are heterogeneous, which is a typical signature of glasses [11].

We find that the freezing temperature derived from the time dependent quantities $\left\langle\Delta r^{2}(t)\right\rangle$ and $\alpha_{2}(t)$ is in agreement with the glass transition temperature $T_{g}$ obtained from the static observables $\Psi_{6}$ and $S(\mathbf{k})$. This should make the experimental observation of the glass phase possible directly from snapshots of particle distributions. The demonstration of a glass phase in a low dimensional monodisperse system with purely repulsive and isotropic interparticle interactions in free space, and its explanation in terms of induced polydispersity, is one of the central results of this work.

In summary, we have demonstrated the existence of glass transitions in monodisperse isotropic systems without disorder. The mechanism for this unusual glass formation has been identified as a consequence of multiscale interaction potentials. While geometrical frustration in typical glass forming materials stems from polydispersity of particles, here, disorder is an effective consequence of frustration in the hopping in the context of cluster crystals and, therefore, a distribution of various cluster sizes. The transition is a two-step process: first, the clusters form, and then, in a second step, they order. It is this second step that shows glassy dynamics due to the effective polydispersity of cluster sizes. One of the physical consequences is that a vortex glass state of matter is possible in clean systems: namely, in thin films of type- 1.5 superconductors. It can also be realized in artificial layered materials that can provide new experimental venues to explore soft-matter models with microscopic control of interactions.

R. D. M., F. M., and G. P. acknowledge support by the European Commission via European Research Council-St Grant ColdSIM (No. 307688) and Rydberg Quantum Simulators, Université de Strasbourg via Laboratoire d'Excellence (LabEx) Nanostructures en Interaction avec leur Environnement (NIE) and Initiative d'Excellence (IdEx), computing time at the High-performance computing - Université de Strasbourg. W. L. acknowledges support by the Austrian Science Fund through Grant No. P 25454N27 and by the Institut fuer Quanteninformation. E. B. acknowledges support from the Goran Gustafsson Foundation and by the Swedish Research Council Grant No. 642-2013-7837.

Note added.-Recently, we became aware of a related work [58] on a cluster glass transition in a model with binary mixtures in three dimensions.

[1] M. P. A. Fisher, Phys. Rev. Lett. 62, 1415 (1989).

[2] D. S. Fisher, M. P. A. Fisher, and D. A. Huse, Phys. Rev. B 43, 130 (1991). 
[3] G. Blatter, M. Feigel'Man, V. Geshkenbein, A. Larkin, and V. M. Vinokur, Rev. Mod. Phys. 66, 1125 (1994).

[4] D. R. Nelson, Defects and Geometry in Condensed Matter Physics (Cambridge University Press, Cambridge, England, 2002).

[5] G. Malescio, J. Phys. Condens. Matter 19, 073101 (2007).

[6] C. N. Likos, Soft Matter 2, 478 (2006).

[7] L. Berthier, A. J. Moreno, and G. Szamel, Phys. Rev. E 82, 060501 (2010).

[8] A. Ikeda and K. Miyazaki, Phys. Rev. Lett. 106, 015701 (2011).

[9] A. Ikeda and K. Miyazaki, J. Chem. Phys. 135, 054901 (2011).

[10] D. Coslovich, M. Bernabei, and A. J. Moreno, J. Chem. Phys. 137, 184904 (2012).

[11] K. Binder and W. Kob, Glassy Materials and Disordered Solids: An Introduction to Their Statistical Mechanics (World Scientific, Singapore, 2011).

[12] V. Moshchalkov, M. Menghini, T. Nishio, Q. H. Chen, A. V. Silhanek, V. H. Dao, L. F. Chibotaru, N. D. Zhigadlo, and J. Karpinski, Phys. Rev. Lett. 102, 117001 (2009).

[13] J. Gutierrez, B. Raes, A. V. Silhanek, L. J. Li, N. D. Zhigadlo, J. Karpinski, J. Tempere, and V. V. Moshchalkov, Phys. Rev. B 85, 094511 (2012).

[14] S. J. Ray, A. S. Gibbs, S. J. Bending, P. J. Curran, E. Babaev, C. Baines, A. P. Mackenzie, and S. L. Lee, Phys. Rev. B 89, 094504 (2014).

[15] I. Kawasaki, I. Watanabe, H. Amitsuka, K. Kunimori, H. Tanida, and Y. Ōnuki, J. Phys. Soc. Jpn. 82, 084713 (2013).

[16] E. Babaev and M. Speight, Phys. Rev. B 72, 180502 (2005).

[17] E. Babaev, J. Carlström, and M. Speight, Phys. Rev. Lett. 105, 067003 (2010).

[18] M. Silaev and E. Babaev, Phys. Rev. B 84, 094515 (2011).

[19] See Supplemental Material at http://link.aps.org/ supplemental/10.1103/PhysRevLett.118.067001 for a derivation of the inter-vortex interaction potential and additional simulations, which includes Refs. [20-35].

[20] M. Silaev and E. Babaev, Phys. Rev. B 85, 134514 (2012).

[21] D. Tilley, Proc. Phys. Soc. London, 84, 573 (1964).

[22] A. Gurevich, Phys. Rev. B 67, 184515 (2003).

[23] A. Gurevich, Physica (Amsterdam) 456C, 160 (2007).

[24] M. E. Zhitomirsky and V.-H. Dao, Phys. Rev. B 69, 054508 (2004).

[25] J. Garaud, M. Silaev, and E. Babaev, arXiv:1601.02227.

[26] E. Babaev, Phys. Rev. Lett. 89, 067001 (2002).

[27] E. Babaev, Nucl. Phys. B686, 397 (2004).

[28] E. Smørgrav, J. Smiseth, E. Babaev, and A. Sudbø, Phys. Rev. Lett. 94, 096401 (2005).

[29] J. Smiseth, E. Smørgrav, E. Babaev, and A. Sudbø, Phys. Rev. B 71, 214509 (2005).

[30] S. B. Chung and S. A. Kivelson, Phys. Rev. B 82, 214512 (2010).

[31] E. Babaev, Phys. Rev. B 79, 104506 (2009).
[32] E. Babaev, J. Carlström, and M. Speight, Phys. Rev. Lett. 105, 067003 (2010).

[33] J. Carlström, E. Babaev, and M. Speight, Phys. Rev. B 83, 174509 (2011).

[34] J. M. Speight, Phys. Rev. D 55, 3830 (1997).

[35] G. Carneiro and E. H. Brandt, Phys. Rev. B 61, 6370 (2000).

[36] J. Pearl, Appl. Phys. Lett. 5, 65 (1964).

[37] M. Tinkham, Introduction to Superconductivity (Dover, New York, 2004).

[38] M. Silaev and E. Babaev, Phys. Rev. B 88, 220504(R) (2013).

[39] J. Bardeen and M. J. Stephen, Phys. Rev. 140, A1197 (1965).

[40] For the larger systems, we used the graphics processing units acceleration provided by the HOOMD-Blue simulation toolkit $[41,42]$.

[41] J. A. Anderson, C. D. Lorenz, and A. Travesset, J. Comp. Physiol. 227, 5342 (2008).

[42] J. Glaser, T. D. Nguyen, J. A. Anderson, P. Liu, F. Spiga, J. A. Millan, D. C. Morse, and S. C. Glotzer, Comput. Phys. Commun. 192, 97 (2015).

[43] F. Maucher, N. Henkel, M. Saffman, W. Krolikowski, S. Skupin, and T. Pohl, Phys. Rev. Lett. 106, 170401 (2011).

[44] B. M. Mladek, D. Gottwald, G. Kahl, M. Neumann, and C. N. Likos, Phys. Rev. Lett. 96, 045701 (2006).

[45] D. Coslovich, L. Strauss, and G. Kahl, Soft Matter 7, 2127 (2011).

[46] M. Montes-Saralegui, A. Nikoubashman, and G. Kahl, J. Phys. Condens. Matter 25, 195101 (2013).

[47] F. Sciortino and E. Zaccarelli, Nature (London) 493, 30 (2013).

[48] C. N. Likos, A. Lang, M. Watzlawek, and H. Lowen, Phys. Rev. E 63, 031206 (2001).

[49] C. N. Likos, B. M. Mladek, D. Gottwald, and G. Kahl, J. Chem. Phys. 126, 224502 (2007).

[50] M. A. Glaser, G. M. Grason, R. D. Kamien, A. Kosmrlj, C. D. Santangelo, and P. Ziherl, Europhys. Lett. 78, 46004 (2007).

[51] R. Díaz-Méndez, F. Mezzacapo, F. Cinti, W. Lechner, and G. Pupillo, Phys. Rev. E 92, 052307 (2015).

[52] E. R. Weeks, J. C. Crocker, A. C. Levitt, A. Schofield, and D. A. Weitz, Science 287, 627 (2000).

[53] H. Tanaka, T. Kawasaki, H. Shintani, and K. Watanabe, Nat. Mater. 9, 324 (2010).

[54] T. E. Markland, J. A. Morrone, B. J. Berne, K. Miyazaki, E. Rabani, and D. R. Reichman, Nat. Phys. 7, 134 (2011).

[55] F. Ebert, P. Keim, and G. Maret, Eur. Phys. J. E 26, 161 (2008).

[56] W. Lechner and P. Zoller, Phys. Rev. Lett. 111, 185306 (2013).

[57] P. Yunker, Z. Zhang, and A. G. Yodh, Phys. Rev. Lett. 104, 015701 (2010).

[58] R. Miyazaki, T. Kawasaki, and K. Miyazaki, Phys. Rev. Lett. 117, 165701 (2016). 